\title{
DYNAMIC CROSSOVER AND LIQUID-LIQUID CRITICAL POINT IN THE TIP5P MODEL OF WATER
}

\author{
LIQUID-LIQUID CRITICAL POINT
}

\author{
P. KUMAR, S. V. BULDYREV, H. E. STANLEY
}

Center for Polymer Studies and Department of Physics, Boston University, Boston, MA 02215 USA Department of Physics, Yeshiva University, 500 West 185th Street, New York, NY 10033 USAA

\begin{abstract}
Water is hypothesized to have a low temperature phase transition line which separates a high density water at high temperatures from a low density water at low temperatures. This negatively sloped first order liquid-liquid phase coexistence line terminates at a critical point known as the liquid-liquid critical point. This critical point is hypothesized to exist in a deeply supercooled region of the phase diagram of water. Recent experiments have given an indirect indication of this phase transition. Neutron scattering and NMR experiments on water confined in hydrophilic nanopores revealed that the dynamics of water changes from the dynamics of a high density liquid (nonArrhenius) at high temperatures to that of a low density liquid (Arrhenius) at low temperatures. Motivated by these experiments we investigated the TIP5P model of water. We find that the dynamic transition appears to be related to crossing the specific heat maxima line (Widom line) which emanates from the liquidliquid critical point. Moreover, we find that water not only undergoes a dynamical change but also structural changes on crossing the Widom line. The structure of the liquid changes from a high density liquid to a low density liquid.
\end{abstract}

Keywords: Liquid-Liquid critical point, fragile-to-strong transition, supercooled water, TIP5P, Widom line. 


\section{Introduction}

Water is the most abundant liquid on earth. This occupies almost 70 percent of the earth's surface and 90 percent of our bodies by volume. It is not only the abundance of water and its importance in biology, atmospheric and engineering sciences that makes it interesting but rather a large set of its unusual properties that makes it unique among liquids. Response functions of water such as isobaric heat capacity, isothermal compressibility etc. seem to diverge as temperature is decreased ${ }^{1,2}$. These and many other unusual properties of water, also referred to as water anomalies, were hypothesized to be the consequence of a first order phase coexistence line between liquids of two different densities which may exist at low temperatures ${ }^{3,4}$. This coexistence line terminates at a critical point which is located in the deep supercooled region of water known as the "no man's land" because it is difficult to do measurements on liquid water in this region as it freezes spontaneously (see Fig. 1). Recent experiments on confined water, where it was possible to avoid freezing of water by confining it to hydrophilic nanopores, indicated that there might be a possibility of a liquid-liquid critical point at low temperatures. Specifically it was found that the dynamics of water changes from non-Arrhenius to Arrehenius behavior upon cooling isobarically. Since non-Arrehenius behavior is a characteristic of high density liquid where the activation energy increases as the temperature decreases and Arrehenius behavior is a characteristic of a low density liquid where the activation energy is a constant, it was speculated that this dynamic transition was somehow associated with the liquid-liquid phase transition. In order to correctly interpret the interesting finding, we need to understand the behavior of the system in the vicinity of a critical point.

By definition, in a first order phase transition, thermodynamic state functions such as density $\rho$ and enthalpy $H$ discontinuously change as we cool the system along a path crossing the equilibrium coexistence line [Fig. $2(\mathrm{a})$, path $\beta]$. In a real experiment, this discontinuous change may not occur at the coexistence line since a substance can remain in a supercooled metastable phase until a limit of stability (a spinodal) is reached ${ }^{1}$ [Fig. 2(b), path $\beta$.

If the system is cooled isobarically along a path above the liquid-gas critical pressure $P_{c}$ [Fig. 2(a), path $\alpha$ ], the state functions continuously change from the values characteristic of a high temperature phase (gas) to those characteristic of a low temperature phase (liquid). The thermodynamic response functions which are the derivatives of the state functions 


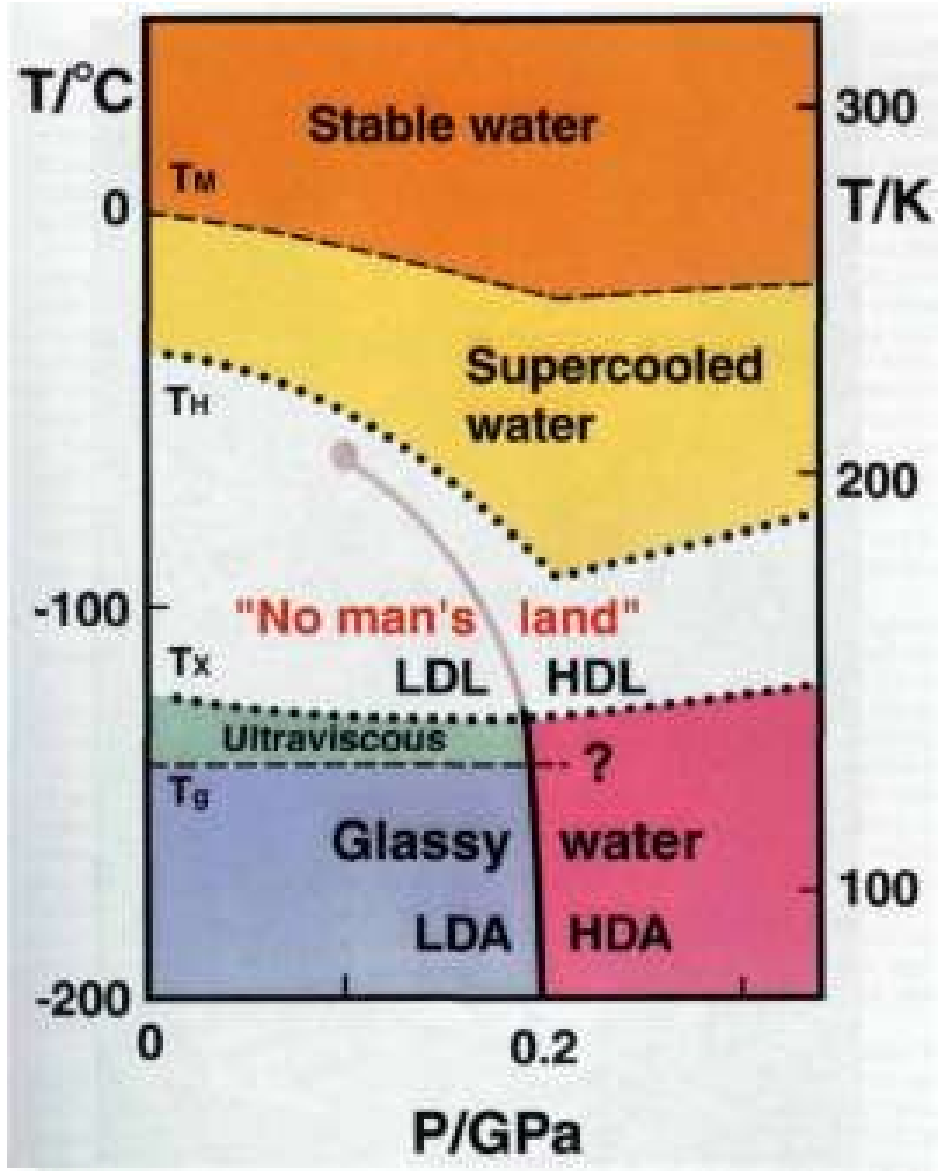

Figure 1: Phase diagram of liquid water. The liquid-liquid critical point lies in the experimentally inaccessible "no man's land." Courtesy of O. Mishima.

with respect to temperature [e.g., isobaric heat capacity $C_{P}=(\partial H / \partial T)_{P}$ ] have maxima at temperatures denoted by $T_{\max }(P)$. Remarkably these maxima are still prominent far above the critical pressure ${ }^{5-8}$, and the values of the response functions at $T_{\max }(P)\left(\right.$ e.g., $C_{P}{ }^{\max }$ ) diverge as the critical point is approached. The lines of the maxima for different response functions asymptotically approach one another as the critical point is approached, since all response functions become expressible in terms of the correlation length. This asymptotic line is sometimes called the Widom line, and is often regarded as an extension of the coexistence line into the "one-phase regime" (Fig. 2). 
If the system is cooled at constant pressure $P_{0}$, and $P_{0}$ is not too far from the critical pressure $P_{c}$, then there are two classes of behavior possible. (i) If $P_{0}>P_{c}$ [Fig. 2(b), path $\alpha$ ] then experimentally-measured quantities will change dramatically but continuously in the vicinity of the Widom line (with huge fluctuations as measured by, e.g., $C_{P}$ ). (ii) If $P_{0}<P_{c}$ [Fig. 2(b), path $\beta$ ], experimentally-measured quantities will change discontinuously if the coexistence line is actually seen. However, the coexistence line can be difficult to detect in a pure system due to metastability, and changes will occur only when the spinodal is approached where the gas phase is no longer stable.

The changes in behavior may include not only static quantities like response functions ${ }^{5-8}$ but also dynamic quantities like diffusivity. In water, a significant change in dynamical properties has been suggested to take place in deeply supercooled states ${ }^{2,9-15}$. Unlike other network forming materials ${ }^{16}$, water behaves as a fragile liquid in the experimentally accessible window ${ }^{2,17,18}$. Based on analogies with other network forming liquids and with the thermodynamic properties of the amorphous forms of water, it has been suggested that, at ambient pressure, liquid water should show a dynamic crossover from fragile behavior at high $T$ to strong behavior at low $T^{14,19-23}$. Using Adam-Gibbs theory ${ }^{24}$, the dynamic crossover in water was related to the $C P^{\max } \operatorname{line}^{11,12,25}$. In this work, we interpret recent experiments on water ${ }^{9-11}$ as arising from the presence of the hypothesized liquid-liquid critical point, which gives rise to a Widom line and an associated fragility transition [Fig. 2(c), path $a$ ].

\section{Methods}

Using molecular dynamics (MD) simulations ${ }^{26}$, we study the TIP5P model of water which accurately represents water behavior at ambient conditions and has a liquid-liquid critical point. This model ${ }^{27}$ treats water as a multiple site rigid body, interacting via electrostatic site-site interactions complemented by a Lennard-Jones potential ${ }^{28}$. We evaluate the loci of maxima of the relevant response functions, compressibility and specific heat, which coincide close to the critical point and give rise to the Widom line. We find evidence that, for both potentials, the dynamic crossover occurs just when the Widom line is crossed. 

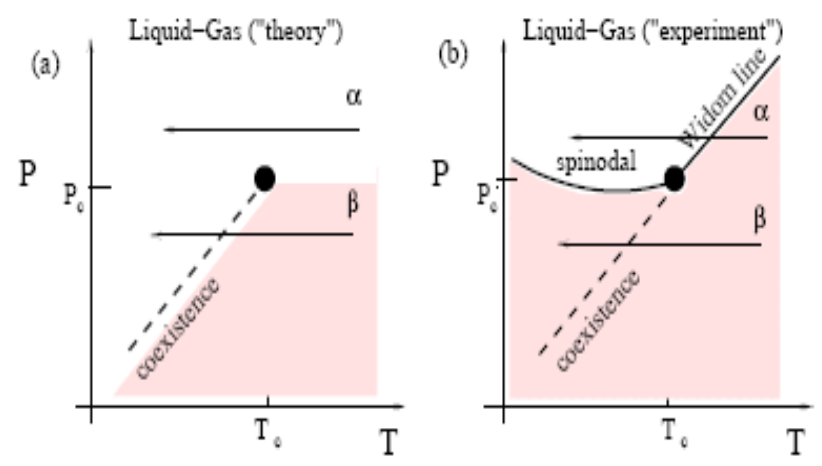

Water

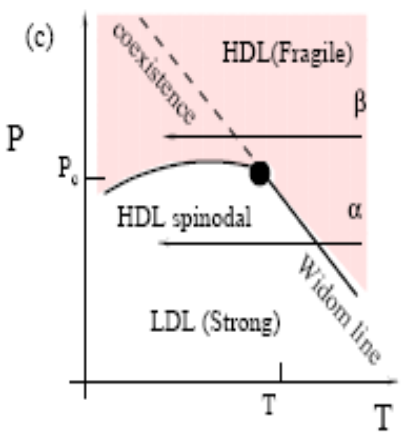

Figure 2: (a) Schematic phase diagram for the critical region associated with a liquidgas critical point. Shown are the two features displaying mathematical singularities, the critical point (closed circles) and the liquid-gas coexistence (bold dashed curve). (b) Same as (a) with the addition of the gas-liquid spinodal and the Widom line. Along the Widom line, thermodynamic response functions have extrema in their $T$ dependence. The path $\alpha$ denotes a path along which the Widom line is crossed. Path $\beta$ denotes a path meeting the coexistence line. (c) A hypothetical phase diagram for water of possible relevance to the recent neutron scattering experiments by Chen et al. ${ }^{9,10}$ on confined water. The liquid-liquid coexistence, which has a negative sloped coexistence line, generates a Widom line which extends below the critical point, suggesting that water may exhibit a fragile-to-strong xs transition for $P<P_{c}$ (path $\alpha$ ), while no dynamic changes will occur above the critical point (path $\beta$ ). Courtesy of L. Xu.

Our results for the TIP5P potential are based on MD simulations of a system of $N=512$ molecules, carried out both in the NVT and NPT ensembles using the techniques described in ${ }^{15,29}$. 


\section{Results}

Figure 3 shows the relevant portion of the $P-T$ phase diagram for the TIP5P model. A liquid-liquid critical point is observed ${ }^{29}$, 30 , from which the Widom line develops. The coexistence curve is negatively sloped, so the Clapeyron equation implies that the high-temperature phase is a high-density liquid (HDL) and the low-temperature phase is a lowdensity liquid (LDL). Fig. 4 shows the $T$ dependence of the diffusion coefficient $D$, evaluated from the long time limit of the mean square displacement along isobars. The isobars crossing the Widom line (path $\alpha$ ) show a crossover (i) from a non-Arrhenius behavior at high $T$ [which can be fit by a power law function $\left.D \sim\left(T-T_{M C T}\right)^{\gamma}\right]$ where $T_{M C T}$ is the crossover temperature predicted by the mode coupling theory ${ }^{31}$, (ii) to an Arrhenius behavior at low $\mathrm{T}$ [which can be described by $\left.D \sim \exp \left(-E_{a} / T\right)\right]$. The crossover between these two functional forms takes place at the Widom line.

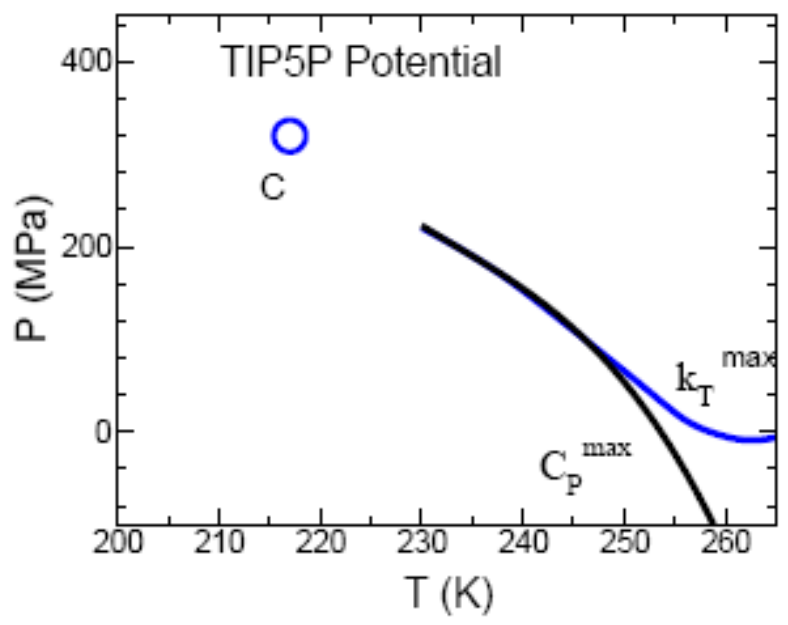

Figure 3: Relevant part of the phase diagram for the TIP5P potential, showing the liquid-liquid critical point $C$ at $P_{c}=320 \mathrm{MPa}$ and $T_{c}=217 \mathrm{~K}$, the line of maximum of isobaric specific heat $C^{\max }$ and the line of maximum of isothermal compressibility $K^{\max }$.

Thus we see that the simulations for TIP5P water model support the connection between the Widom line and the dynamic fragility crossover. Crossing the Widom line, not only gives a dynamic crossover but also the structural changes where water goes from a less ordered, "high-densitylike" water at high temperatures to more ordered, "low-density-like" water at low temperatures. In Fig. 5, we show the oxygen-oxygen pair correlation 
functions $g(r)$ corresponding to two temperatures, one above the Widom line and another below the Widom line. Above the Widom line at $\mathrm{T}=250 \mathrm{~K}$, water is less ordered as seen from the smaller first and second peaks of $g(r)$, while below the Widom line at $\mathrm{T}=230 \mathrm{~K}$, water is more ordered, reflected in the increase in the amplitude of the first and the second peaks of $g(r)$. The area between the first and the second peak of $g(r)$ decreases as the Widom line is crossed, suggesting that the number of bifurcated bonds decreases and hence fifth neighbors

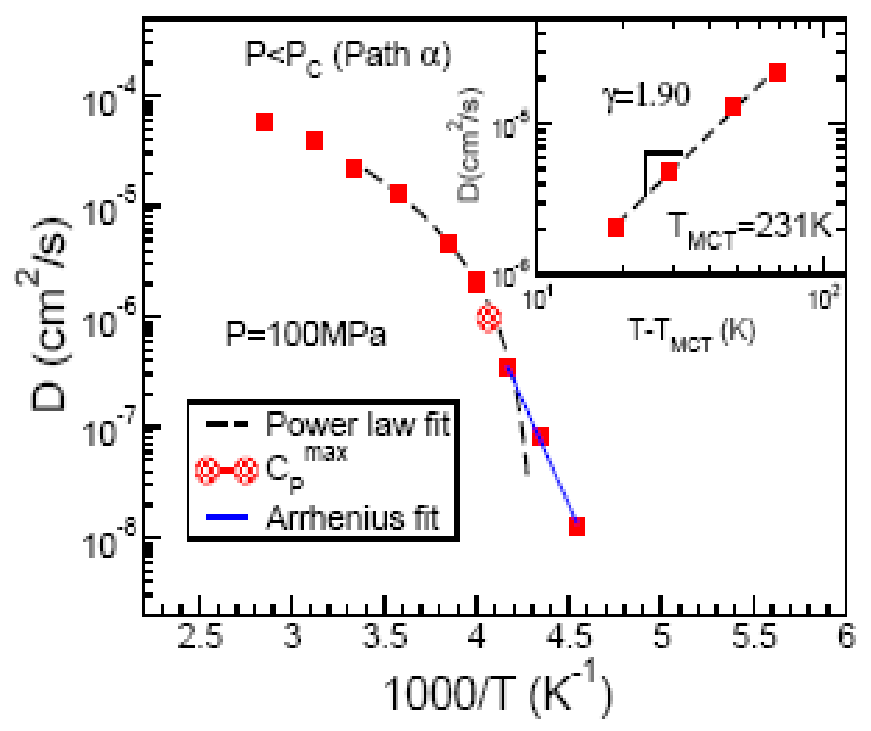

Figure 4: At high temperatures, $D$ behaves like that of a non-Arrhenius liquid and can be fit by $D \sim\left(T-T_{M C T}\right)^{r}$ (also shown in the inset) where $T_{M C T}=231 \mathrm{~K}$ and $\gamma=1.90$, while at low temperatures the dynamic behavior appears to change to that of a liquid where $D$ is Arrhenius. 


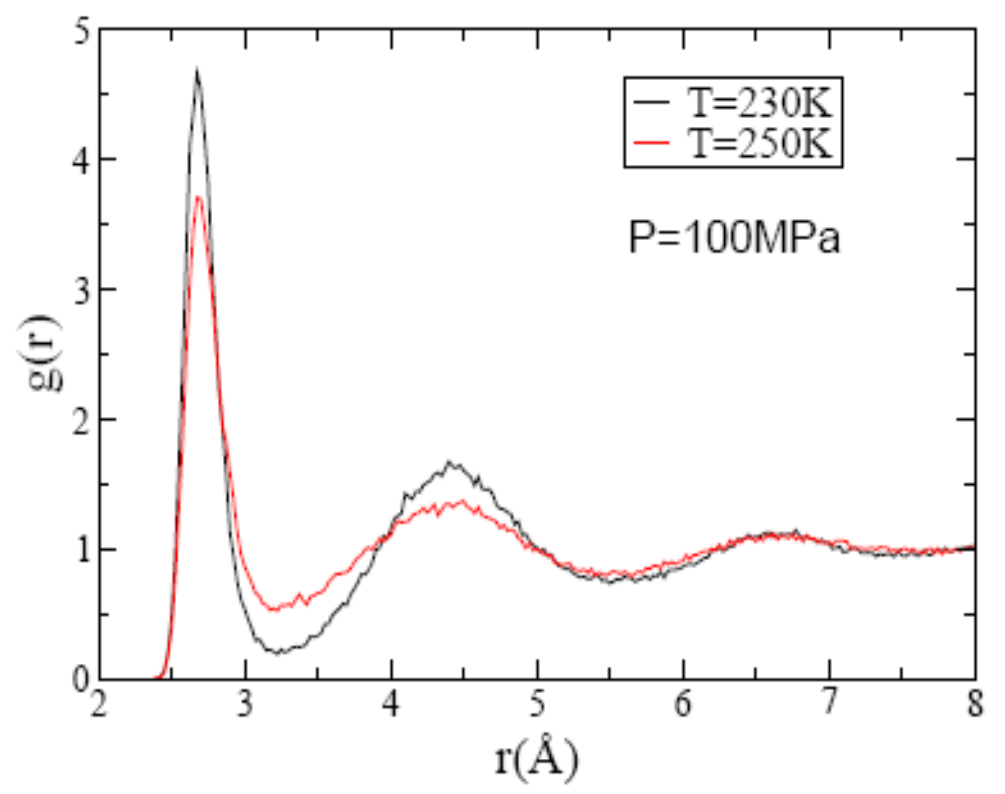

Figure 5: Oxygen-oxygen pair correlation function above and below the Widom line at $\mathrm{P}=100 \mathrm{MPa}$. Above the Widom line, water is less ordered while upon crossing the Widom line, water becomes more ordered

(which is a feature of high density water) also decreases upon crossing the Widom line from the high temperature side to the low temperature side. Figure 6 shows the structure factor of water on two sides of the Widom line at $\mathrm{P}=100 \mathrm{MPa}$. The structure of the liquid on high temperature side of the Widom line is less tetrahedral compared to the structure of the liquid on the low temperature side of the Widom line. In Fig. 7, we show the corresponding hydrogen bonds formed by the water molecules with a hydrogen bondlength cut-off distance of $0.3 \mathrm{~nm}$ and angle width of 10 degrees. For the temperature below the Widom line water is more structured locally and hence more hydrogen bonds with a strict constraint of the hydrogen-bond angle are formed compared to water above the Widom line temperature. 


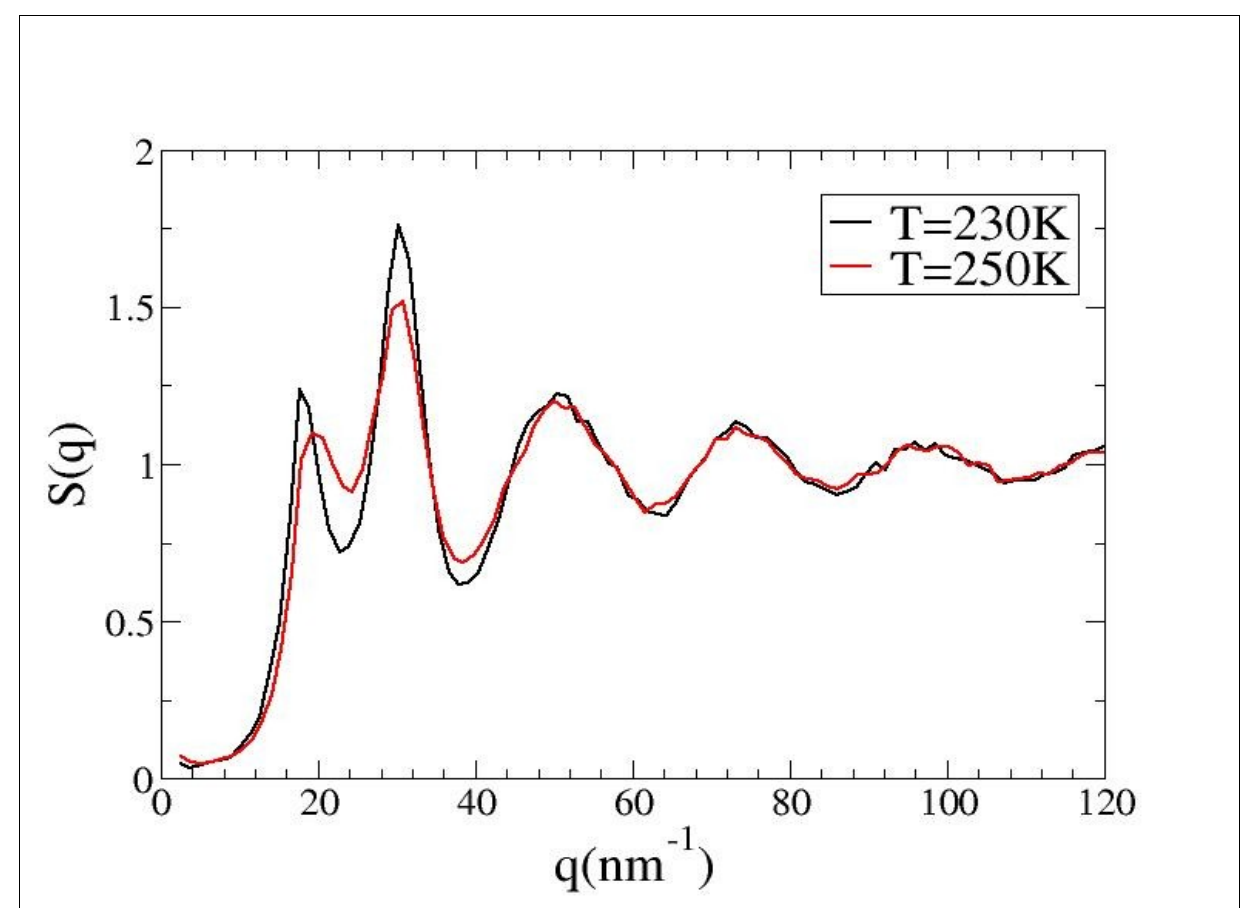

Figure 6: Structural changes on crossing the Widom line at $P=100 \mathrm{MPa}$. (a) Structure factor $S(q)$ above the Widom line (see Fig. 5). (b) Structure factor $S(q)$ below the Widom line. Tetrahedrality of the water molecules increases dramatically upon crossing the Widom indicated by the a sharp increase in the first and second peaks of the structure factor. 

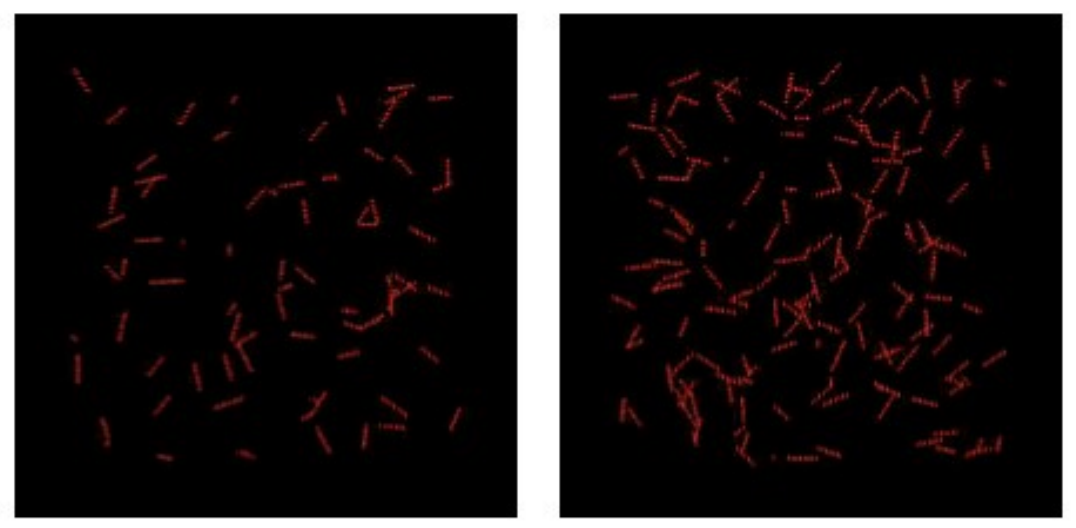

Figure 7: Structural changes on crossing the Widom line at $P=100 \mathrm{MPa}$. (a) Hydrogen bonds above the Widom line (see Fig. 5).(b) Hydrogen bonds below the Widom line. Number of the hydgrogen bonds with strict tetrahedral structures increases upon crossing the Widom line.

\section{Discussion and summary}

Our findings are consistent with the possibility that the observed dynamic crossover along path $a$ is related to the behavior of $C_{P}$, suggesting that enthalpy or entropy fluctuations may have a strong influence on the dynamic properties ${ }^{11,12,32}$. Indeed, as the thermodynamic properties change from the high-temperature side of the Widom line to the low-temperature side, $(\partial S / \partial T) p=C_{P} / T>0$ implies that the entropy must decrease. The entropy decrease is most pronounced at the Widom line when $C_{P}=C_{P}{ }^{\max }$. Since the configurational part of the entropy, $\mathrm{S}_{\text {conf, }}$ makes the major contribution to $S$, we expect that $S_{\text {conf }}$ also decreases sharply on crossing the Widom line.

$D$ changes from non-Arrhenius ("fragile") to Arrhenius ("strong") behavior, while the structural and thermodynamic properties change from those resembling HDL to those resembling LDL, due to the negative slope of the Widom line.

In summary, our results are consistent with the experimental observation in confined water of (i) a fragility transition for $P<P_{c}{ }^{9,10}$, and (ii) a peak in $C_{P}$ upon cooling water at atmospheric pressure ${ }^{33}$. Thus our work offers a plausible interpretation of the results of Ref. ${ }^{9-11}$ as supporting the existence of a hypothesized liquid-liquid critical point. 


\section{Acknowledgements}

We thank C A. Angell, S.-H. Chen, G. Franzese, J. M. H. Levelt Sengers, L. Liu, M. G. Mazza, P. H. Poole, S. Sastry, F. Sciortino, F. W. Starr, B. Widom, L. Xu, and Z. Yan for helpful discussions and NSF grant CHE 0096892 for support. We also thank the Boston University Computation Center, Yeshiva University Office of Academic Affairs, and StFX hpcLAB for allocation of CPU time.

\section{References}

1. Debenedetti, P. G. \& Stanley, H. E. (2003) Physics Today 56 (6), 4046.

2. Debenedetti, P. G. (2003) J. Phys.: Condens. Matter 15, R1669R1726.

3. Mishima, O. \& Stanley, H. E. (1998) Nature 392, 164-168.

4. Poole, P. H., Sciortino, F., Essmann, U. \& Stanley, H. E. (1992) Nature 360, 324-328.

5. Anisimov, M. A., Sengers, J. V. \& Levelt Sengers, J. M. H. (2004) in Aqueous System at Elevated Temperatures and Pressures: Physical Chemistry in Water, Stream and Hydrothermal Solutions, ed. Palmer, D. A., Fernandez-Prini, R. \& Harvey, A. H. (Elsevier, Amsterdam).

6. Levelt, J.M.H. (1958) in Measurements of the compressibility of argon in the gaseous and liquid phase, Ph.D. Thesis (University of Amsterdam, Van Gorkum \& Co., Assen).

7. Michels, A., Levelt, J.M.H. \& Wolkers, G. (1958) Physica 24, 769794.

8. Michels, A., Levelt, J.M.H. \& de Graaff, W. (1958) Physica 24, 659671.

9. Liu, L., Chen, S.-H., Faraone, A., Yen, C.-W \& Mou, C.-Y (2005) Phys. Rev. Lett. 95, 117802.

10. Faraone, A., Liu, L, Mou, C.-Y, Yen, C.-W. \& Chen, S.-H (2004) J. Chem. Phys. 121, 10843- 10846.

11. Mallamace, F. \& Chen, S. -H. (2006) J. Chem. Phys. (in press)

12. Xu, L., Kumar, P., Buldyrev, S. V., Chen, S.-H., Poole, P, Sciortino, F., and Stanley, H. E. (2005) Proc. Nat. Acad. Sci. 102, 16558.

13. Chen, S.-H. et. al. (submitted to Proc. Nat. Acad. Sci.) (2006) .

14. Kumar P., Xu, L., Yan, Z., Mazza, M., Buldyrev, S.V., Chen, S.-H., Sastry, S., and Stanley, H. E., (2006) (submitted) .

15. Scala, A., Starr, F. W., La Nave, E., Sciortino, F. \& Stanley, H. E. (2000) Nature 406, 166-169. 
16. Starr, F.W., Angell, C A. \& Stanley, H. E. (2003)Physica A 323, 5166.

17. Angell, C. A. (2004) Ann. Rev. Phys. Chem. 55, 559-583.

18. Angell, C. A. (1993) J. Phys. Chem. 97, 6339-6341.

19. Kumar, P., Buldyrev, S. V., Starr, F. W., Giovambattista, N. \& Stanley, H. E. (2005) Phys.Rev. E 72, 051503.

20. Horbach, J. \& Kob, W. (1999) Phys. Rev. B 60, 3169-3181.

21. Lang, E. W. \& Ludemann, H. D. (2004) Angew Chem. Intl. Ed. Engl. 21, 315-329.

22. Prielmeier, F. X., Lang, E. W, Speedy, R. J., Ludemann, H. D. \& Bunsenges., Ber. (1987) Phys. Rev. Lett. 59, 1128-1131.

23. Ito, K., Moynihan, C. T. \& Angell, C. A. (1999) Nature 398, $492-$ 495.

24. Jagla, E. A. (1999) J. Chem. Phys. 111, 8980-8986.

25. Jagla, E. A. (1999) J. Phys.: Condens. Matter 11, 10251-10258.

26. Jagla, E. A. (2001) Phys. Rev. E 63, 061509.

27. Tanaka, H. (2003) J. Phys.: Condens. Matter 15, L703-L711 .

28. Poole, P. H., Saika-Voivod, I. \& Sciortino, F. (2005) J. Phys.: Condens. Matter 17, L431- L437.

29. Rapaport, D. C. (1995) in The Art of Molecular Dynamics Simulation (Cambridge University Press, Cambridge).

30. Mahoney, M. W \& Jorgensen, W. L. (2000) J. Chem. Phys. 112, 8910-8922.

31. Stillinger, F. H. \& Rahman, A. (1972) J. Chem. Phys. 57, 1281-1292.

32. Yamada, M., Mossa, S., Stanley, H. E. \& Sciortino, F (2002) Phys. Rev. Lett. 88, 195701.

33. Paschek, D. (2005) Phys. Rev. Lett. 94, 217802.

34. Gotze, W. (1991) in Liquids, Freezing, and Glass Transition, edited by J. P. Hansen, D. Levesque, and J. Zinn-Justin (NorthHolland, Amsterdam), pp. 287.

35. Saika-Voivod, I., Poole, P. H. \& Sciortino, F. (2001)Nature 412, 514-517.

36. Maruyama, S., Wakabayashi, K. \& Oguni, M. (2004) AIP Conf. Proc. 708, 675-676. 
